\title{
Sequential clinical and histopathological changes in collagenous and lymphocytic colitis over time
}

\author{
Beth H Shaz ${ }^{1}$, Sarathchandra I Reddy ${ }^{2}$, Gamze Ayata ${ }^{1, *}$, Tom Brien ${ }^{3}$, Francis A Farraye ${ }^{2, \dagger}$, \\ Donald A Antonioli ${ }^{1}$, Robert D Odze ${ }^{3}$ and Helen H Wang ${ }^{1}$ \\ ${ }^{1}$ Department of Pathology, Beth Israel Deaconess Medical Center, Boston, MA, USA; ${ }^{2}$ Department of \\ Medicine and ${ }^{3}$ Department of Pathology, Brigham and Women's Hospital, Harvard Medical School, Boston, \\ $M A, U S A$
}

\begin{abstract}
We conducted this retrospective study to evaluate the relationship between symptoms, histological findings, and treatment of collagenous (CC) and lymphocytic colitis (LC). We identified 19 CC and 12 LC patients having multiple colonoscopic procedures with colonic biopsies during their course of illness. A detailed histological review of all biopsies was performed. Clinical history, including symptoms and medications, was obtained in 25 of the 31 patients and was correlated with their histological findings. In all, $25 \%$ of the CC patients and $50 \%$ of the LC patients who had biopsies prior to their definitive diagnosis had the pathognomonic histological features on their prior biopsies to some extent (but were not recognized by the pathologists); however, these features were more pronounced on the biopsies from the procedure that established the diagnosis. Nonetheless, 10 of 12 such patients with clinical data available had symptoms and were being treated at the time of prior biopsies. Assessment of the relationship among histological, clinical and therapeutic data showed no association between symptoms or histological findings and treatment with any medication. In summary, in this sample of CC and LC patients, symptoms often precede fully developed histological features. No change in symptoms or histological findings was found to be associated with medication.
\end{abstract}

Modern Pathology (2004) 17, 395-401, advance online publication, 20 February 2004; doi:10.1038/modpathol.3800070

Keywords: microscopic; lymphocytic; collagenous; colitis; sequential; symptoms; histology

'Microscopic colitis' refers to a clinicopathologic entity consisting of chronic watery diarrhea combined with a normal appearance of the colonic mucosa on endoscopy and distinct abnormalities detected on histological examination of mucosal biopsy specimens. ${ }^{1}$ Two forms of microscopic colitis have been identified: collagenous colitis (CC), ${ }^{2}$ characterized by thickening and irregularity of the subepithelial collagen layer and by an increased number of inflammatory cells in the lamina propria and epithelium, and lymphocytic colitis (LC), ${ }^{3,4}$ which has a similar degree of lamina propria inflammation

Correspondence: Dr BH Shaz, MD, Department of Pathology, Beth Israel Deaconess Medical Center, 330 Brookline Avenue, Boston, MA 02215, USA. E-mail: bshaz@caregroup.harvard.edu

Presented in part at the 90th annual meeting of the United States and Canadian Academy of Pathology in Atlanta, GA, March 5, 2001.

${ }^{*}$ Current affiliation: Department of Pathology, UMass Memorial Medical Center, Worcester, MA

Current affiliation: Section of Gastroenterology, Boston Medical Center, Boston, MA, USA.

Received 21 April 2003; revised 29 October 2003; accepted 10

December 2003; published online 20 February 2004 as CC, but a more prominent intraepithelial lymphocytic component and a normal thickness of the subepithelial collagen layer. These two forms of microscopic colitis are being diagnosed with increasing frequency; they now account for $10 \%$ of cases evaluated for chronic nonbloody diarrhea that are not associated with human immunodeficiency virus (HIV) infection at tertiary medical centers. ${ }^{5}$

Since the etiology of these conditions is unknown, therapy has been largely empiric. Furthermore, the natural history of these entities has not been well defined. Thus, in the absence of randomized controlled studies, the effect of any therapy is difficult, if not impossible, to evaluate. We, therefore, conducted this retrospective study to evaluate sequential clinical and histological findings in patients with CC or LC in order to correlate symptoms with histological findings as well as with treatment.

\section{Materials and methods}

All patients with a diagnosis of lymphocytic, collagenous, or microscopic colitis within a specific 
time period were identified from the Brigham and Women's Hospital (BWH) (1992-1999) and the Beth Israel Deaconess Medical Center (BIDMC) (19951999). Approval was obtained from Internal Review Board on studies of human subjects in both institutions. Patients who had endoscopic procedures with biopsies performed before and/or subsequent to the endoscopic procedure at which the diagnosis of LC or CC was initially established were included in this study. The diagnostic mucosal biopsies and all prior or subsequent biopsies were collected and the date of the biopsy, age and sex of the patient, and the original diagnoses were recorded.

Two pathologists reviewed hematoxylin- and eosin-stained slides from each institution (GA and DA from BIDMC and TB and RO from BWH) for a variety of histological features without knowledge of the patients' clinical or endoscopic findings. The features evaluated included: crypt architectural irregularity (crypt distortion characterized by the presence of $\geq 2$ branched crypts at the base of the mucosa), crypt atrophy (defined as crypts that were separated from the muscularis mucosae by inflammatory cells), crypt inflammation (defined as the presence of neutrophils within the crypt epithelium, or within the crypt lumen), surface erosion or ulceration, Paneth cell metaplasia (defined as the presence of Paneth cells in biopsies located distal to the transverse colon), intraepithelial lymphocyte (IEL) count per 100 surface enterocytes in the most concentrated area (not over lymphoid nodules), basally located lymphoid aggregates in the lamina propria, diffuse mixed inflammation in the lamina propria (defined as a mixture of lymphocytes, plasma cells, neutrophils, and eosinophils throughout the full thickness of the lamina propria), thickness of the subepithelial collagen layer measured with the use of an Olympus type calibrated eye-piece ( $\times 10$ magnification) on an Olympus microscope (Olympus $\mathrm{B} \times 45$ with Uplan objective) and recorded in microns, as well as the nature of interface between subepithelial collagen layer and lamina propria (smooth vs irregular).

In addition to the presence of increased inflammation in the lamina propria, the specific histopathologic diagnosis of CC was based on a focal or continuous thickening of the subepithelial collagen layer $(10 \mu \mathrm{m}$ or more), damage to the surface epithelium with or without increased IEL infiltration. The histopathologic diagnosis of LC was based on increased surface IEL (>20/100 enterocytes), surface epithelial degeneration, but a normal subepithelial collagen layer.

Clinical data, including medical history, symptoms and signs, such as abdominal pain; diarrhea (frequency, time of occurrence, and quality-loose, watery, or containing mucus); endoscopic features; and treatment at the time of each procedure, were extracted from the patients' medical records for correlation.
The clinical and histological findings in the prior and follow-up biopsies were compared to those in the diagnostic biopsies using the sign rank test. They were correlated with one another with MannWhitney test or Spearman's $r$. All statistical analyses were performed with STATA software, release 6 (Stata Press, College Station, TX, USA).

\section{Results}

\section{Patient Population}

Of 150 consecutive patients with a confirmed diagnosis of CC or LC, 31 patients (19 CC and 12 LC patients) had prior and/or subsequent biopsies in addition to their initial diagnostic biopsies. Of the 19 CC patients, 10 had previous, seven had followup, and two had both previous and follow-up biopsies. Of the 12 LC patients, six had prior and six had subsequent biopsies. The average age of the patients at diagnosis was 63 years (range 34-86 years). All 19 CC patients were female, whereas two of the 12 LC patients were male. The number of prior endoscopic procedures was either 1 or 2, with 1-6 (median 2) specimens obtained from 1-6 (median 2) locations per procedure. The distribution of biopsy locations in the colon from the diagnostic procedures appeared to be more even than that from the prior procedures, which included more from the distal colon. For the diagnostic procedures, 12, 16, $20,18,18$, and $14 \%$ were from cecum, ascending colon, transverse colon, descending colon, sigmoid colon, and rectum, respectively, with $2 \%$ from unspecified location. This is in contrast to the prior procedures; $8,8,11,16,24$, and $32 \%$ are from cecum, ascending colon, transverse colon, descending colon, sigmoid colon, and rectum, respectively. The diagnostic procedure seemed to have involved better sampling (range of 1-11 (median 5) specimens and 1-6 (median 4) locations, $P<0.001$ ). The number of follow-up endoscopic procedures ranged from 1 to 4 with 1 to 10 (median 4) specimens from 1 to 6 locations (median 3) per procedure. No significant difference was noted in sampling between the diagnostic and the follow-up procedures $(P>0.15)$. The median of the time interval between the first and last procedures for each patient was 24 months (range $0.2-87$ months). There was no significant difference in the median time interval between that from the prior to the diagnostic procedure (20 months) and that from the diagnostic to the follow-up procedure (24 months).

\section{Sequential Histological Findings}

Histological findings were compared between prior and diagnostic, and diagnostic and follow-up biopsies. When multiple biopsies were taken in one procedure, the most advanced finding of all biopsies was used for each of the features examined. Since 
some patients had more than one prior or follow-up biopsy, there are more procedures than patients in these comparisons. The diagnostic features of LC (maximum IEL count > 20/100 enterocytes) and CC (increased subepithelial collagen deposition to $>10 \mu \mathrm{m}$ ), as well as nonspecific increase in lamina propria inflammation were more apparent on the diagnostic biopsies (Tables 1 and 2) than on the prior biopsies. The statistically significant differences between the prior and diagnostic biopsies in CC (Table 1) included increased percentage in the diagnostic biopsies of Paneth cell metaplasia, increased lamina propria inflammation, and irregular subepithelial collagen interface. There was also increased average subepithelial collagen thickness in the diagnostic biopsies. The only statistically significant difference between the diagnostic and follow-up biopsies in CC was a decrease in average subepithelial collagen thickness on follow-up (Table 1). No statistically significant differences were found in LC patients, either between the prior and the diagnostic or between the diagnostic and the follow-up biopsies (Table 2).

\section{Clinical Data}

Clinical data were obtained for 25 of the 31 patients. The past medical history was significant for gastrointestinal diseases in 10 of the 25 patients. Three patients had colon cancer, one patient had celiac disease and a previously diagnosed CC (LC on our biopsies), and one patient had a previous diagnosis of LC, ulcerative colitis, and eosinophilic gastritis. One had diverticulitis and one had Clostridium difficile infection. One patient carried the diagnosis of chronic active colitis and one had a history of indeterminate colitis.

When more than one prior or follow-up procedures were conducted, the clinical and histological data at the procedure that were closest in time to that of the diagnostic procedure were used for

Table 1 Histological findings ${ }^{\mathrm{a}}$ in collagenous colitis with prior and/or follow-up biopsies

\begin{tabular}{|c|c|c|c|c|}
\hline & Prior & Diagnostic & Diagnostic & Follow-up \\
\hline Total number of patients & 12 & 12 & 9 & 9 \\
\hline Total number of procedures & 15 & 12 & 9 & 13 \\
\hline Architecture abnormality (\%) & 0 & 8 & 11 & 15 \\
\hline Crypt inflammation (\%) & 33 & 33 & 33 & 38 \\
\hline Surface epithelial degeneration or inflammation (\%) & 54 & 67 & 78 & 83 \\
\hline Surface erosion (\%) & 13 & 0 & 11 & 8 \\
\hline Paneth cell metaplasia (\%) & $13^{\mathrm{b}}$ & $64^{\mathrm{b}}$ & 67 & 54 \\
\hline IEL count > 20/100 enterocytes (\%) & 31 & 67 & 33 & 62 \\
\hline IEL distribution (both surface and crypt) (\%) & 62 & 83 & 78 & 92 \\
\hline Lymphoid nodules > 1 (\%) & 53 & 75 & 78 & 38 \\
\hline Lamina propria mononuclear cell inflammation (\%) & $60^{\mathrm{b}}$ & $100^{\mathrm{b}}$ & 100 & 92 \\
\hline Subepithelial collagen thickness $(\mu \mathrm{m})$ & $5^{b}$ & $15^{\mathrm{b}}$ & $19^{\mathrm{b}}$ & $14^{\mathrm{b}}$ \\
\hline Diffuse (\%) & 25 & 83 & 78 & 44 \\
\hline Irregular epithelial and LP interface (\%) & $50^{\mathrm{b}}$ & $100^{\mathrm{b}}$ & 100 & 100 \\
\hline Subepithelial collagen thickness, rectum $(\mu \mathrm{m})$ & $3^{\mathrm{b}}$ & $11^{\mathrm{b}}$ & 17 & 11 \\
\hline
\end{tabular}

${ }^{a}$ The most serious finding of all biopsies in the procedure was used for each of the features examined.

${ }^{\mathrm{b}} \mathrm{P}<0.05$ : prior vs diagnostic biopsies and follow-up vs diagnostic biopsies.

Table 2 Histological findings ${ }^{\mathrm{a}}$ in lymphocytic colitis with prior or follow-up biopsies

\begin{tabular}{|c|c|c|c|c|}
\hline & Prior & Diagnostic & Diagnostic & Follow-up \\
\hline Total number of patients & 6 & 6 & 6 & 6 \\
\hline Total number of procedures & 6 & 6 & 6 & 7 \\
\hline Architecture abnormality (\%) & 0 & 0 & 0 & 0 \\
\hline Crypt inflammation (\%) & 50 & 50 & 67 & 29 \\
\hline Surface epithelial degeneration or inflammation (\%) & 50 & 83 & 83 & 80 \\
\hline Surface erosion $(\%)$ & 0 & 0 & 0 & 0 \\
\hline Paneth cell metaplasia (\%) & 0 & 20 & 0 & 0 \\
\hline IEL count > 20/100 enterocytes (\%) & 50 & 100 & 100 & 57 \\
\hline IEL count > 20/100 enterocytes, rectum (\%) & 33 & 0 & 60 & 100 \\
\hline IEL distribution (both surface and crypt) (\%) & 33 & 100 & 100 & 85 \\
\hline Lymphoid nodules > $1(\%)$ & 50 & 83 & 100 & 100 \\
\hline Lamina propria inflammation (\%) & 50 & 100 & 100 & 71 \\
\hline Subepithelial collagen thickness $(\mu \mathrm{m})$ & 3 & 3 & 3 & 3 \\
\hline
\end{tabular}

\footnotetext{
${ }^{\mathrm{a}}$ The most serious finding of all biopsies in the procedure was used for each of the features examined.
} 

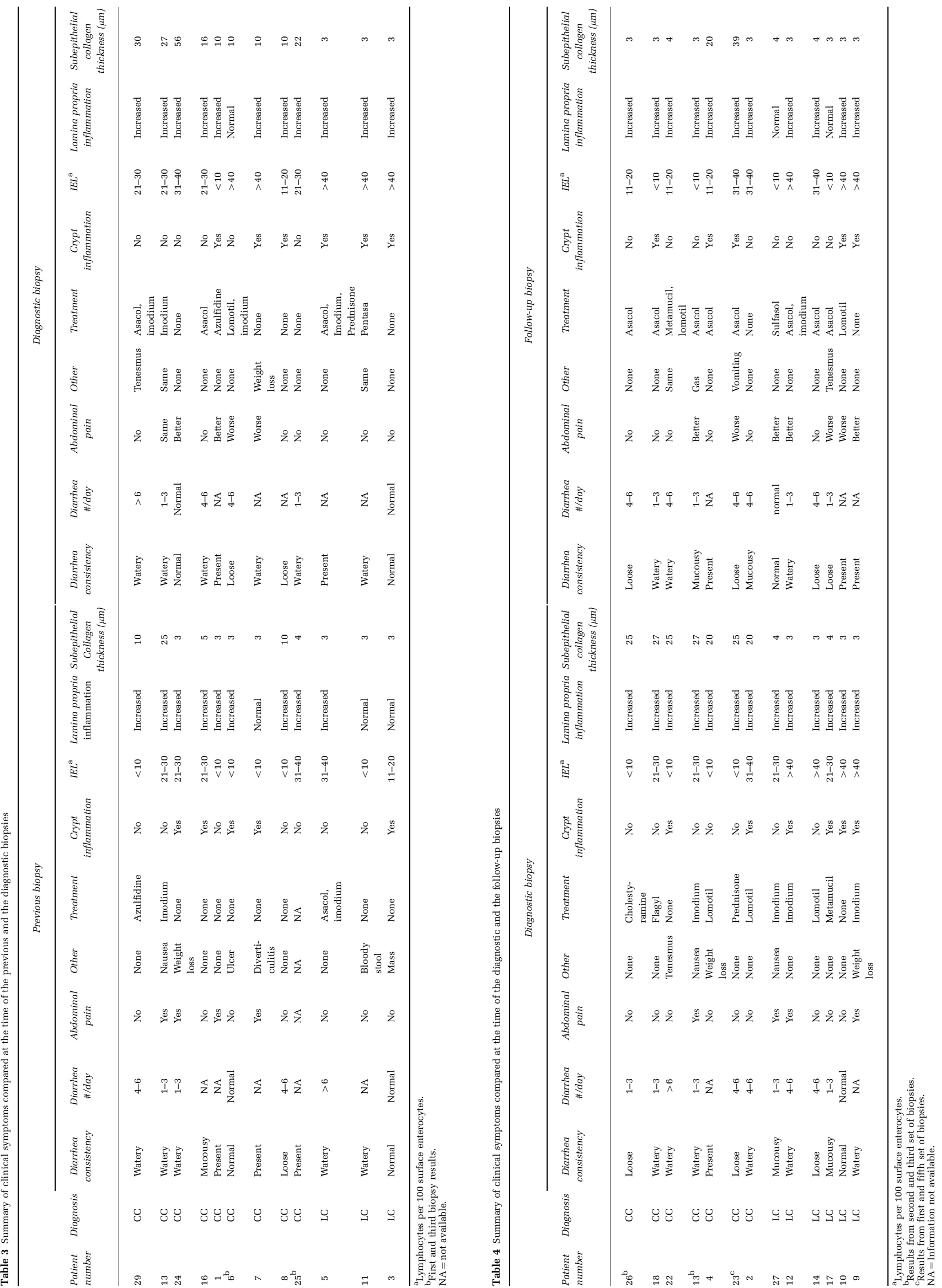
comparison with the data at diagnosis and inclusion in Tables 3 and 4 . Of the 12 patients with previous biopsies and clinical data (nine CC and three LC), all but two patients had diarrhea at the time of previous procedure (Table 3). Symptoms were the same or worse over the course in eight patients, better in three patients; one patient (\#3), who underwent colonoscopy for a mass lesion (colon cancer), never had symptoms at the time of previous or diagnostic procedure. All but one of the 13 patients with follow-up biopsies and clinical data (seven CC and six LC) had diarrhea at the time of the diagnostic procedure (Table 4). The only patient who was an exception developed diarrhea subsequently.

Three of the 12 patients who had prior biopsies were receiving treatment (azulfidine in one, imodium in two, and asacol in one) at the time of previous biopsy and four additional patients were on medications at the time of diagnostic biopsy (asacol in three, azulfidine in one, imodium in four, lomotil in one, pentasa in one, and prednisone in one) (Table 3). Only one patient was on the same medication between these two procedures and some patients were on multiple medications. Of the 13 patients who had follow-up biopsies, 11 were receiving treatment at the time of the diagnostic procedure (flagyl in one, imodium in four, lomotil in three, metamucil in one, prednisone in one, and cholestyramine in one) (Table 4). All but two patients were still on treatment (asacol in eight, lomotil in two, metamucil in one, sulfasalazine in one, and imodium in one) at the time of follow-up (Table 4). No patients were on identical regimens between these two procedures.

As shown in Table 3, there was no statistically significant change in the consistency and frequency of bowel movements or the presence of abdominal pain between the time of the prior procedure and the time of the procedure when the diagnosis was established $(P>0.05$ with sign rank test). The symptoms were the same in most patients and approximately the same number of patients had improvement and worsening of their symptoms. The diagnostic features (intraepithelial lymphocytosis or increased subepithelial collagen deposition) were, however, significantly more apparent in the diagnostic biopsies (with $P=0.02$ and 0.006 , respectively, with sign rank test). Table 4 shows that there was no overall significant change in any of the symptoms between the time when the diagnosis was established and the time of follow-up. The only significant change in the histological findings was a decreased thickness of collagen layer in the CC patients $(P=0.05)$.

\section{Correlation of Treatment, Symptoms, Histological Findings, and Time Interval}

We combined the data in Tables 3 and 4 and considered the earlier procedure (whether previous

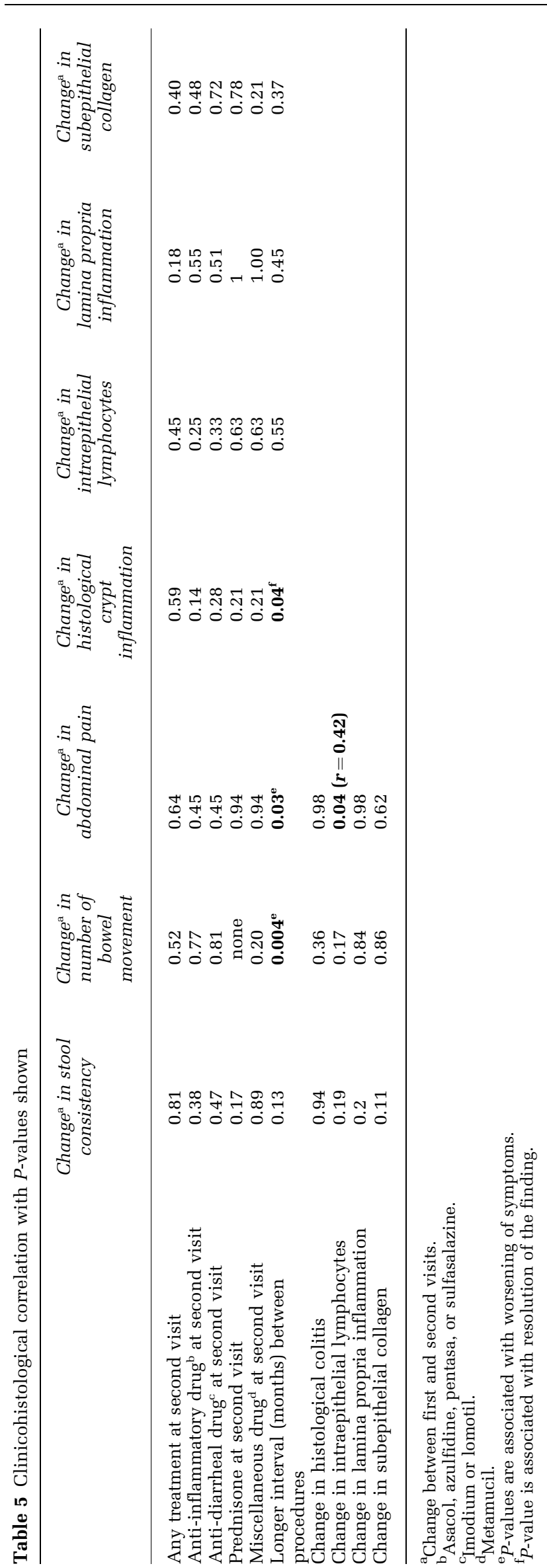

Modern Pathology (2004) 17, 395-401 
or diagnostic) as the first visit, and the later procedure (whether diagnostic or follow-up) as the second visit. Table 5 shows the correlation between changes in various symptoms and histological findings with different treatments at the time of the second visit, the correlation between changes of symptoms and changes in histological findings, as well as the changes in symptoms and histological findings with the time interval between the two visits. There was no association found between any of the symptoms or histological findings and the use of any medication at the second visit. A significant association was noted between an improvement of abdominal pain and a reduction of IEL (with Spearmen's $r=0.42, P=0.04$ ). The length of the time interval between the first and second visit was significantly associated with three features. The number of bowel movements and abdominal pain were more likely to become worse with longer interval between the two visits ( $P$-value of 0.004 and 0.03 , respectively), whereas histological crypt inflammation was more likely to decrease with longer interval between the two visits $(P=0.04)$ (Table 5).

\section{Discussion}

The entity of 'microscopic colitis' has been recognized for over 20 years; however, its natural history remains unclear. Although sequential histological evaluations and follow-up of clinical presentations have been reported for collagenous colitis ${ }^{6}$ and lymphocytic colitis, $^{7}$ the relationship between changes in symptoms and histological findings, as well as the relationship between changes in symptoms and histological findings with treatment, is still undetermined. Our study included both patients with biopsies before the diagnosis of microscopic colitis was established and patients with follow-up biopsies after the diagnosis was made. Furthermore, in addition to histological findings, information on symptoms and treatment was obtained on most of the study patients at the time of each procedure. It should be pointed out, however, that our study subjects are not a random sample of all patients with CC and LC. These patients could very well have had two or more endoscopic procedures in part because they had persistent symptoms refractory to treatment. This impression could be deduced from the fact that most patients in our study changed treatment between the first (either the previous or diagnostic) and the second (either the diagnostic or follow-up) procedure (Tables 3 and 4), and the fact that most of the CC and LC patients at the two participating institutions (119 of $150=79 \%$ ) had only a single endoscopic procedure during the study period.

A comparison of the biopsies taken before the diagnosis was made, and those that yielded a diagnosis for the first time of either collagenous or lymphocytic colitis showed that many patients had epithelial and lamina propria inflammation in the earlier biopsies (Tables 1 and 2). Although in a few patients (\#5, 13, and 25 in Table 3) a definitive diagnosis could have been made but was missed in the earlier biopsies, the diagnostic features of collagenous and lymphocytic colitis were either absent or insufficiently developed for a definitive diagnosis in most of these earlier biopsies. In spite of the less than diagnostic histological features, most of the patients already had diarrhea at the time of the earlier biopsies. While this clinicohistological discrepancy probably reflects sampling errors in the prior procedure, it cannot be excluded that CC and LC evolve over time, and that symptoms may precede the full-blown histological picture. A second explanation may be that the nonspecific mucosal inflammation present in the earlier biopsies, rather than the intraepithelial lymphocytosis and/or subepithelial collagen deposition, is the cause of diarrhea. ${ }^{8}$ In fact, it was recently shown that the degree of lamina propria inflammation was a predictor of response to treatment in CC patients. ${ }^{9}$ Still another explanation is that multiple independent factors in addition to lamina propria inflammation and subepithelial collagen deposition may be the mechanisms of diarrhea in CC. ${ }^{10}$ Of particular interest in our results is the presence of Paneth cell metaplasia in $13 \%$ of the earlier biopsies from patients with CC and in over $50 \%$ of the biopsies that were diagnostic of CC and the follow-up biopsies of CC patients (Table 1). This finding is not unique to this small group of microscopic colitis patients. Similar findings have been noted in the large group of microscopic colitis patients from which this subgroup was derived. ${ }^{11}$ This finding lends support to the notion that CC is a chronic condition.

In contrast to the comparison of histological findings between prior and diagnostic biopsies, the comparison between the diagnostic and follow-up biopsies showed hardly any differences. The only significant change was a decrease in the average subepithelial collagen deposition in the follow-up biopsies of CC patients compared to that in the diagnostic biopsies (14 vs $19 \mu \mathrm{m}$ ); however, it was still increased in the follow-up biopsies $(14 \mu \mathrm{m})$. The other histological findings remained unchanged (Tables 1 and 2). Carpenter et al. ${ }^{6}$ reported histological resolution in eight of 14 CC patients after an average of 14 months of follow-up, and Mullhaupt et $a l^{7}$ described histological 'normalization' in 25 of 27 (93\%) LC patients after an average of 38 months of follow-up. The fact that histological abnormalities persisted in most of our patients, especially those with CC, may indicate that our series represents a selective group of microscopic colitis patients, as speculated above, or a shorter follow-up interval (median of 24 months).

Tables 3 and 4 show that no overall significant differences in symptoms were observed between the 
first (either the previous or the diagnostic) and the second (either the diagnostic or the follow-up) procedure $(P>0.05$ with sign rank test). In the two series of longitudinal studies of CC and LC patients cited earlier, ${ }^{6,7}$ even in patients with histological regression, the symptoms did not subside concomitantly in all the patients.

When data from Tables 3 and 4 are combined and changes in symptoms and histological findings between first and second visits are correlated with each other and with treatment, some interesting results emerged. The only significant correlation between a change in symptoms and a change in histological findings is between a reduction in IELs and an improvement in abdominal pain (Table 5 , $P=0.04$ and Spearman's $\rho=0.42$ ). As far as we are aware, this is the first report of such an association. However, no association was found between the use of any medication and symptoms or histological findings. Although microscopic colitis has not traditionally been characterized by abdominal pain, a high percentage of microscopic colitis patients were recently found to have abdominal pain. ${ }^{12,13}$

Analysis of the time interval between the first and second visits and changes in symptoms and histological findings revealed a significant correlation between changes in number of bowel movements, abdominal pain, and histological crypt inflammation with length of interval (Table 5). It is interesting that an increase in bowel movements and abdominal pain was associated with long interval, reinforcing the notion that the patients might have undergone another endoscopic procedure because of this. The crypt inflammation might have improved over time because the histology went more from acute to chronic changes. Although crypt inflammation has not been considered an integral part of these chronic colitides, it was recently reported and examined along with other inflammatory bowel disease-like morphologic features in our study of a larger group of microscopic colitis patients. ${ }^{11}$

In summary, in a series of 31 microscopic colitis patients who had two or more colonoscopic procedures with detailed clinical data of symptoms and treatment associated with each procedure in 25 of them, we found that many patients had mucosal inflammation and diarrhea without the histological hallmarks of CC and LC before the diagnostic subepithelial collagen deposition and intraepithelial lymphocytosis became well developed. This suggests that diarrhea may be associated with nonspecific mucosal inflammation or other factors rather than specifically with intraepithelial lymphocytosis or collagen deposition. In addition, we found no association between treatment and change in symptoms or histological findings. This study is limited by potential selection bias of subjects with more severe microscopic colitis due to its retrospective nature and relatively small number of patients in each category of treatment.

\section{References}

1 Schiller LR. Microscopic colitis syndrome: lymphocytic colitis and collagenous colitis. Semin Gastrointest Dis 1999;10:145-155.

2 Lindstrom CG. 'Collagenous colitis' with watery diarrhoea-a new entity? Pathol Eur 1976;11:87-89.

3 Read NW, Krejs GJ, Read MG, et al. Chronic diarrhea of unknown origin. Gastroenterology 1980;78:264-271.

4 Lazenby AJ, Yardley JH, Giardiello FM, et al. Lymphocytic ('microscopic') colitis: a comparative histopathologic study with particular reference to collagenous colitis. Hum Pathol 1989;20:18-28.

5 Fine KD, Seidel RH, Do K. The prevalence, anatomic distribution, and diagnosis of colonic causes of chronic diarrhea. Gastrointest Endosc 2000;51:318-326.

6 Carpenter HA, Tremaine WJ, Batts KP, et al. Sequential histologic evaluations in collagenous colitis. Correlations with disease behavior and sampling strategy. Dig Dis Sci 1992;37:1903-1909.

7 Mullhaupt B, Guller U, Anabitarte M, et al. Lymphocytic colitis: clinical presentation and long term course. Gut 1998;43:629-633.

8 Schiller LR. Pathophysiology and treatment of microscopic-colitis syndrome. Lancet 2000;355:1198-1199.

9 Abdo A, Raboud J, Freeman HJ, et al. Clinical and histological predictors of response to medical therapy in collagenous colitis. Am J Gastroenterol 2002;97: 1164-1168.

10 Burgel N, Bojarski C, Mankertz J, et al. Mechanisms of diarrhea in collagenous colitis. Gastroenterology 2002;123:433-443.

11 Ayata G, Ithamukkala S, Sapp H, et al. Prevalence and significance of inflammatory bowel disease-like morphologic features in collagenous and lymphocytic colitis. Am J Surg Pathol 2002;26:1414-1423.

12 Pardi DS, Smyrk TC, Tremaine WJ, et al. Microscopic colitis: a review. Am J Gastroenterol 2002;97:794-802.

13 Fernandez-Banares F, Salas A, Esteve M, et al. Collagenous and lymphocytic colitis. Evaluation of clinical and histological features, response to treatment, and long-term follow-up. Am J Gastroenterol 2003;98:340-347. 